\title{
A Functional Recovery after Resuscitative Thoracotomy for Traumatic Cardiac Tamponade in an 87 Year-0ld
}

\author{
Lynn Model and Bishwajit Bhattacharya* \\ Department of surgery, Yale school of medicine, New Haven, Connecticut, USA
}

Received: November 27, 2015; Accepted: December 04, 2015; Published: December 09, 2015

*Corresponding author: Bishwajit Bhattacharya, Department of surgery, Yale school of medicine, New Haven, Connecticut, USA, Tel: (203) 605-8011;

Email-bishwajit.bhattacharya@yale.edu

\section{Introduction}

Indications for emergent resuscitative thoracotomy have been intensely debated over the past 40 years. Conclusions drawn from composite retrospective reviews have determined that resuscitative thoracotomy for blunt chest trauma with recent loss of vital signs has overall very poor outcomes. Conversely, this procedure is recommended in patients presenting with penetrating chest injuries and recent loss of vital signs, with metaanalysis and large case series reporting 14.6-19.8\% survival rates $(1,2)$. Particularly in cases of singular penetrating cardiac injuries, there are reports of up to $45.5 \%$ survival in select patients (i.e. singular stab wounds with signs of life on presentation), versus $0 \%$ survival in patients with multiple penetrating injuries and no signs of life on presentation (3). Of interest, the average patient in these reviews is a young male in their mid-twenties, with no patients above 40 years old.

Thoracotomy in elective lung resections is itself a morbid procedure that carries a reported morbidity rate of $23-43.6 \%$ and a mortality rate of $1.2-11.3 \%$. These are significantly higher morbidity and mortality rates than in similar procedures that are performed thoracoscopically, pointing out the morbidity of the thoracotomy approach itself. These rates trend up as age of the patient increases, with one study of thoracotomy in octogenarians reporting a complication rate of $63 \%$ (4-8). It follows that thoracotomies performed in the emergency department, often with haste and with poor sterile technique, carry even higher morbidity and mortality rates; notwithstanding the initial traumatic insult that led to the need for such an intervention.

We present the case of an 87 year-old man who underwent an emergent resuscitative thoracotomy for cardiac tamponade and survived. The tamponade was caused by a cardiac injury from chest tube placement for hemo-pneumothorax.

\section{Case}

An 87-year-old man with a was brought to the emergency room by a friend after a fall 1 week prior and a second fall 1 day prior, where his initial complaints were of pain in his left hip, neck, and abdomen as well as generally feeling unwell. At the time of triage his vital signs were all within normal limits; blood pressure of 109/61, heart rate of 73, and oxygen saturation of $93 \%$ on room air. His past medical history was significant for only hypertension. A chest x-ray and cat scan of the abdomen/pelvis demonstrated multiple left-sided rib fractures with resultant moderate pleural effusion and pneumothorax and no intraabdominal pathology. A left chest tube was placed, returning $700 \mathrm{ml}$ of blood. The patient became immediately hypotensive to a nadir blood pressure of 55/37. He was emergently intubated and had an internal jugular triple lumen placed. Repeat labs demonstrated a hematocrit drop from 31 to $21 \mathrm{mg} / \mathrm{dl}$. He was started on a dopamine drip and given 2 units of uncrossmatched packed RBC. A Repeat cat scan of the chest, abdomen, and pelvis was performed that demonstrated a new moderate pericardial effusion. His condition temporarily stabilized with these measures and he was then urgently transferred to a nearby tertiary care center.

On initial trauma re-evaluation his blood pressure was $118 / 71$, heart rate was 74 , and his oxygen saturation was $100 \%$ on assist-control ventilation. An endotracheal tube was in place, but the patient was alert and interactive with eyes open, responding to questions appropriately with head nods. His cardiac exam was significant for a rub and his lung exam was notable for decreased breath sounds on the left side, where a chest tube was sutured in place with approximately one liter of blood in the canister. A repeat chest x-ray upon transfer demonstrated near-complete opacification of the left hemithorax as well as a left chest tube that terminated at the lung apex. A focused-assessment with sonography for trauma (FAST) exam was performed which was positive for a notable pericardial effusion. While undergoing the remainder of the trauma evaluation, the patient's blood pressure dropped from normal range to barely palpable. Emergent left thoracotomy was performed at the bedside. This allowed visualization of a tense, purple pericardium that was immediately opened in a cephalad-caudad direction and large amounts of blood clot were evacuated from around the heart. No obvious source of bleeding was identified at this time. Initially the heart was stagnant, but almost immediately returned rhythm after removal of the surrounding clot. Blood pressure returned with systolics in the 80 's. The patient was then brought to the operating room where exploration of the left chest and heart 
revealed a contusion and $1 \mathrm{~cm}$ laceration to the left ventricle that was not actively bleeding. This was repaired with a plegetted 4-0 prolene suture. All superficial chest wall muscular bleeding was controlled, the left chest cavity was thoroughly irrigated, and 3 chest tubes were placed and put to suction. The patient remained intubated and was subsequently transferred to the Surgical Intensive Care Unit.

Initially the patient required fluid and pressor support with norepinephrine which was weaned off over hospital days 2 through 4. He was continued on broad-spectrum prophylactic antibiotics (pipercillin-tazobactam) for 8 days given concern for poor sterility of his initial surgical procedure. He underwent daily spontaneous breathing trials and was extubated on hospital day 7. His chest tubes were removed on hospital day 8 and hospital day 13. Postoperatively he developed atrial fibrillation but this reversed to sinus rhythm with aggressive electrolyte repletion. He was discharge to a short-term rehabilitation center on hospital day 15.

\section{Discussion}

Cardiac tamponade caused by chest-tube placement is a rarely reported complication $(9,10)$. Chest-tube insertions are routinely performed in the emergency setting, with other complications of insertion that are often referenced including tube malposition or kinking, lung injury, nerve and vascular injuries, and residual pneumothorax. Rarer injuries include those to the heart, esophagus, pulmonary artery or thoracic duct (11). Cardiac tamponade may be managed by pericardiocentesis or open surgical drainage. Surgical options in the elective patient with slow-onset symptoms include subxyphoid and trans-thoracic pericardial windows (12). In the acute setting, resuscitative thoracotomy provides quick access to the pericardium and the ability to manually remove all clot. It also allows for access the entirety of the heart to potentially stop the source of bleeding.

Thoracotomy is a particularly morbid procedure in the elderly, and resuscitative thoracotomy is known to be an innately morbid procedure. Despite this, in the patient with acute tamponade with hemodynamic compromise it should be considered, regardless of age. This is especially true in patients with a singular 'stab' wound to the heart as it was in this case, and as it has been reported previously in a considerably younger patient population.

\section{References}

1. Rhee PM, Acosta J, Bridgeman A, Wang D, Jordan M, Rich N. Survival after emergency department thoracotomy: review of published data from the past 25 years. J Am Coll Surg. 2000; 190(3):288-98.

2. Seamon MJ, Chovanes J, Fox N, Green R, Manis G, Tsiotsias G. The use of emergency department thoracotomy for traumatic cardiopulmonary arrest. Injury. 2012; 43(9):1355-61. doi: 10.1016/j. injury.2012.04.011.

3. Seamon MJ, Shiroff AM, Franco M, Stawicki SP, Molina EJ, Gaughan JP. Emergency department thoracotomy for penetrating injuries of the heart and great vessels: an appraisal of 283 consecutive cases from two urban trauma centers. J Trauma. 2009; 67(6):1250-7; discussion 1257-8. doi: 10.1097/TA.0b013e3181c3fef9.

4. Port JL, Mirza FM, Lee PC, Paul S, Stiles BM, Altorki NK. Lobectomy in octogenarians with non-small cell lung cancer: ramifications of increasing life expectancy and the benefits of minimally invasive surgery. Ann Thorac Surg. 2011; 92(6):1951-7. doi: 10.1016/j. athoracsur.2011.06.082.

5. Cattaneo SM, Park BJ, Wilton AS, Seshan VE, Bains MS, Downey RJ. Use of video-assisted thoracic surgery for lobectomy in the elderly results in fewer complications. Ann Thorac Surg. 2008; 85(1):231-5.

6. Whitson BA, D'Cunha J, Andrade RS, Kelly RF, Groth SS, Wu B, Thoracoscopic versus thoracotomy approaches to lobectomy: differential impairment of cellular immunity. Ann Thorac Surg. 2008; 86(6):1735-44. doi: 10.1016/j.athoracsur.2008.07.001.

7. Phillips JD, Merkow RP, Sherman KL, DeCamp MM, Bentrem DJ, Bilimoria KY. Factors affecting selection of operative approach and subsequent short-term outcomes after anatomic resection for lung cancer. J Am Coll Surg. 2012; 215(2):206-15. doi: 10.1016/j. jamcollsurg.2012.04.016.

8. Papiashvilli M, Stav D, Cyjon A, Haitov Z, Gofman V, Bar I. Lobectomy for non-small cell lung cancer: differences in morbidity and mortality between thoracotomy and thoracoscopy. Innovations (Phila). 2012; 7(1):15-22. doi: 10.1097/IMI.0b013e3182566221.

9. Hesselink DA, Van Der Klooster JM, Bac EH, Scheffer MG, Brouwers JW. Cardiac tamponade secondary to chest tube placement. Eur J Emerg Med. 2001; 8(3):237-9.

10. Quak JM, Szatmari A, van den Anker JN. Cardiac tamponade in a preterm neonate secondary to a chest tube. Acta Paediatr. 1993; 82(5):490-1.

11. Kesieme EB, Dongo A, Ezemba N, Irekpita E, Jebbin N, Kesieme C. Tube thoracostomy: complications and its management. Pulm Med. 2012; 2012:256878. doi: 10.1155/2012/256878.

12. Grecu L. Cardiac tamponade. Int Anesthesiol Clin. 2012; 50(2):59-77. doi: 10.1097/AIA.0b013e318254053e. 